Cartographie critique des usages et des significations attribués au concept d'ethnogenèse dans les Globalization Studies

Lionel Obadia

\title{
CpenEdition
}

Journals

Édition électronique

URL : https://journals.openedition.org/pa/1890

DOI : $10.4000 /$ pa. 1890

ISSN : 2273-0362

Éditeur

Université Lumière Lyon 2

Édition imprimée

Date de publication : 1 janvier 2006

Pagination : 7-27

ISBN : $1643-7706$

ISSN : 1634-7706

Référence électronique

Lionel Obadia, «Cartographie critique des usages et des significations attribués au concept

d'ethnogenèse dans les Globalization Studies », Parcours anthropologiques [En ligne], 6| 2006, mis en ligne le 06 juillet 2021, consulté le 21 juillet 2021. URL : http://journals.openedition.org/pa/1890 ; DOI : https://doi.org/10.4000/pa.1890 


\section{Cartographie critique des usages et des SIGNIFICATIONS ATTRIBUÉS AU CONCEPT D'ETHNOGENÈSE dans les Globalization Studies}

Qu'elle soit énoncée de manière explicite ou de manière plus confidentielle dans les développements théoriques contemporains des Globalization Studies, la notion d'ethnogenèse s'y impose actuellement comme un opérateur théorique de plus en plus sollicité, mais qui émerge en ordre dispersé et dans des contextes d'usage sensiblement différents. Plus que d'une émergence, c'est de la renaissance d'un concept dont il est ici question, concept d'ailleurs quelque peu oublié ou négligé par les sciences sociales occidentales, en vertu de l'étiolement des paradigmes sur lesquels elle prenait appui, en l'occurrence, l'évolutionnisme et l'essentialisation des ethnies. Son retour timide mais significatif sur le devant de la scène intellectuelle ou, du moins, sa réhabilitation dans certains secteurs des sciences de l'homme et de la société, ne manque pas d'interroger les raisons de cette redécouverte, ainsi que ses nouveaux champs d'application.

Cet article propose une cartographie, malheureusement très panoramique, des significations et des usages d'une notion (formulée sous la forme du terme conceptuel ou suggérée à travers l'usage d'autres vocables) qui connaît des prolongements qui s'éloignent quelque peu de ses formulations initiales - et de ses persistances - dans le contexte des sciences sociales et historiques soviétiques. Par-delà le repérage lexical, qui procède d'une géographie à plat, il s'agit ainsi de retracer l'histoire du concept, et d'en dégager les transformations de sens dans la profondeur diachronique. En procédant à une lecture des continuités et des discontinuités des propriétés conceptuelles et des usages dans quelques œuvres récentes, on s'efforcera donc ici de saisir les filiations et les enjeux des redéfinitions de l'ethnogénèse. La notion reste à l'évidence tributaire des problématiques plus générales de l'ethnicité, et, partant, de l'étude des labellisations, des catégories du Soi (collectif) inventées ou réinventées, et donc dans l'analyse, plus globale, donc des identités collectives et de leur dynamique. C'est pourquoi on conservera à l'esprit que les trois concepts identité ethnicité - ethnogenèse (auquel il faut ajouter celui d'autochtonie qui ne pourra être questionné ici) sont indissociables, même s'ils ont connu des postérités très différentes - l'ethnogenèse étant le dernier à pénétrer le 
champ théorique ouvert par les deux premiers. Avec pour objectif final de restituer et d'examiner, de manière critique, le rôle des Globalization Studies dans la résurgence du concept d'ethnogenèse.

\section{SuRfACE et USAGES : ENTRE HISTOIRE ET ESSENCE}

L'ethnogenèse - comme substantif, rarement utilisé sous des formes adjectivées - est fondée sur un "noyau » de signification, celui d'une définition de base qui se résume comme suit :la manière dont se constitue un peuple dans l'histoire et dans sa singularité. C'est un invariant de ces utilisations dans la littérature scientifique. Cette définition minimale mais extensive et admettant un haut degré de généralité, ne spécifie en définitive que peu un terme dont l'opérationnalité demeure floue : à l'évidence, toutes les sciences ayant un peu affaire à l'histoire sont susceptibles de référer à une ethnogenèse. Mais toutes les sciences historiques n'ont néanmoins pas utilisé - à plus forte raison théorisé - ce concept. La notion d'ethnogenèse est également loin d'être nouvelle, et les phénomènes dont elle est supposée rendre compte aussi anciens que les cultures et les civilisations elles-mêmes. Elle a en outre a été formulée bien avant celle de mondialisation, et ses usages identifiés, bien avant que les Globalization Studies ne s'en saisissent, sont quelque peu différents de ceux qu'en font ces dernières. La portée opératoire du concept - qui traduit une vision historique des sociétés humaines, plus qu'une conception fonctionnelle ou structurale de ces dernières - mérite donc que l'on se penche plus avant sur les domaines ou les problématiques dans lesquels son usage est commun.

Sur le plan empirique, la notion d'ethnogenèse recouvre généralement les modalités (conditionnées par des circonstances économiques, des configurations culturelles, des morphologies sociales, ou des agencements écologiques particuliers...) par lesquelles se fonde un collectif humain au fil de l'histoire globale, et de sa trajectoire particulière. Si de très larges domaines des Sciences de l'Homme pourraient aisément se retrouver dans une telle perspective, c'est plutôt du côté des publications qui mentionnent explicitement la notion, en titre ou dans le corps du texte, que l'intérêt se porte. La surprise vient assurément du constat que l'ethnogenèse ne fait souvent l'objet d'aucune précision conceptuelle. Pour ne prendre qu'un exemple, puisé dans la littérature récente (ou un peu moins), en ethnologie, Thomas Turner a ainsi intitulé l'un de ses articles « Ethnogenesis in Zaire » (1993); il y rediscute les variations ethnonymiques des Luba-Kasai du Zaïre au fil de l'histoire, et surtout des forces politiques en présence (locales et coloniales) qui ont conjointement contribué à ces changements. Les contradictions en matière d'exactitude des identités affichées par les Luba-Kasai, ou déterminées par les ethnologues, qui opposent les spécialistes de la région, rendent la reconstitution de l'histoire des Luba des plus malaisées. Ainsi l'ethnogenèse relève-t-elle ici d'abord et surtout d'une question académique : celle de la vérité historique de l'identité des Luba-Kasai. L'ethnogenèse offre égale- 
ment à l'analyse une profondeur diachronique qui permet d'identifier les contextes et enjeux des cristallisations identitaires qui ont, par exemple, généré l'antagonisme (et le bain de sang qui s'en est suivi) entre les Hutu et les Tutsi du Rwanda (Taylor, 2004). Mais l'ethnologie n'est pas la seule science humaine à convoquer le concept d'ethnogenèse. On le retrouve également dans les travaux d'archéologie, lorsqu'ils retracent par exemple la formation des identités slaves (Curta, 2001), dans l'histoire médiévale, qui, nourrie des théories de l'ethnicité, entreprend de reconstituer les modalités de constitution des " peuples » à travers les modes de pensée et les modalités d'écriture de l'histoire (Hoppenbrowers, 2006), ou enfin dans l'histoire antique, lorsqu'elle pense l'ethnogenèse comme la fixation d'identités distinctes entre les peuples des temps bibliques (Killbrow, 2005). Aucun de ces travaux, il convient de le mentionner, ne discute réellement la notion, qui ne se voit attribuer ici qu'un sens nominal.

\section{IDENTITÉ ET ETHNICITÉ DANS LES SCIENCES SOCIALES CONTEMPORAINES}

Le sens de la notion d'ethnogenèse ne peut se comprendre sans référer à d'autres concepts qui configurent son réseau sémantique, l'identité et l'ethnicité, et le plan de la réalité humaine sur lequel elles se manifestent, l'histoire des sociétés humaines. Dans tous les cas, l'ethnogenèse a une histoire et celle-ci, buissonnante et polymorphe, débute (sous nos latitudes), avec les débats sur l'ethnie. Cela fait longtemps, déjà, que les développements critiques sur l'ethnie ont scellé le sort des vues essentialistes ou primordialistes qui lui donnaient forme et essence : l'ethnie n'est plus, ou seulement pour un nombre désormais très réduit d'anthropologues ou d'historiens, une entité admettant suffisamment de consistance sociologique et historique pour faire l'objet d'une étude scientifique, à la manière des sciences naturelles étudiant des objets autonomes des conditions de leur production. Depuis le chapitre consacré aux " groupes ethniques » par Max Weber dans son œuvre majeure, Économie et Société(1971 pour la version française), qui disséquait les modalités de construction collective de l'identification ethnique, la consistance de l'ethnie s'en est trouvée singulièrement mise à mal, et une large partie de la sociologie s'est désormais ralliée à ces conceptions (Streiff-Fenart \& Poutignat, 1995). De son côté, l'anthropologie a également procédé (et achevé) la dé-réification de l'ethnie. Dès les années 1950 et 1960, des chercheurs comme Edmund Leach, Michael Moerman ou encore Frederick Barth (tous ethnologues) ont travaillé successivement à montrer le caractère stratégique, dynamique et situé des labellisations ethniques - et l'on ne peut que s'étonner qu'un livre de référence sur la question de l'ethnique (Streiff \& Poutignat, 1995) invite encore les ethnologues à ne pas céder à la " naïveté » de croire en l'existence de l'ethnie. Au concept d'ethnie a succédé - un point de clarification que le lecteur familier avec ces questions trouverait accessoire - le concept d'ethnicité, qui s'appuie sur des conceptions nettement plus dynamiques, et qui a connu une incontestable popularité depuis que Glazer et Moynihan lui ont consacré un volume qui fait date pour ces 
questions (1975 pour la version française). Depuis les années 1960, le volume de publications (articles, livres, journaux spécialisés...) consacrées à l'ethnicité n'a cessé de s'accroître. En France, l'ethnicité s'est imposée par exemple dans des domaines d'étude où elle avait auparavant été peu usitée, comme dans la sociologie des religions : le pluralisme religieux contemporain s'explique ainsi en termes de pluralité " ethnique ». Sans qu'il soit toujours fait mention de l'habituelle " modernité » à laquelle on associe généralement la diversité confessionnelle, ni encore à la mondialisation, l'ethnicité se résume finalement aux revendications d'une singularité exprimée par des groupements religieux (entre eux ou en rapport à la société globale), en particulier ceux qui sont qualifiés de minoritaires (Lamine, 2005). Le problème, c'est que, sous cet angle, toute forme de différence affirmée peut alors être qualifiée d'" ethnique » et le terme même d'ethnicité risque fort de devenir " a passing theoretical fad - a tropical, not to say trendy, subject that consumes a lot of attention and journal space " (Drummond, 1983). C'est dans le contexte de ces progrès rapides, mais controversés, de la thématique de l'ethnicité qu'a récemment ressurgi le concept d'ethnogenèse.

\section{RACINES： L'ETHNOGRAPHIE SOVIÉTIQUE}

C'est dans l'etnografya soviétique - science taxinomique sur le plan descriptif, évolutionniste sur celui de la théorie - que le concept d'ethnogenèse a initialement connu ses plus amples développements : Shirokogoroff, le premier, a théorisé, en 1963, le concept clef de l'anthropologie russe, l'ethnos, unité ethnique de base, inscrite dans une histoire, et dont les propriétés sont dépendantes de l'économie et du politique. Concept singulier qui distingue la tradition soviétique des autres grandes anthropologies nationales (Dragaze, 1980), il a été - et reste encore - au cœur des traditions anthropologiques à l'Est (Bonte, Izard, Lenclud, 2002).

Yu Bromley a rappelé, à l'occasion de la confrontation entre les anthropologies occidentales et soviétiques, que l'objet (object) et le sujet (subject-matter) de l'etnografya est l'« ethnos ", une unité de population non réductible à une nation, une " race", une société ou une culture, mais qui présente néanmoins des traits distinctifs « objectifs » (comme des traits de psychologie collective) (Bromley, 1980 : 153). Le modèle d'analyse des ethnos est celui de l'évolutionnisme, mesurant leurs variations de forme sur une échelle historique qui va de l'« archaïque " au "développé » (Bromley, 1980). Bromley rappelle ainsi que les principes fondamentaux (paradoxaux, pour l'anthropologie inspirée du constructivisme et antiévolutionniste à l'Ouest), que « [les ethnos] sont des systèmes dynamiques. Ainsi, une des tâches les plus importantes de l'ethnographie est d'étudier les changements qui s'opèrent en leur sein, c'est-à-dire les processus ethniques. Alors que par le passé, ces processus étaient relativement lents, au final, ils ont mené à la disparition de certaines unités ethniques et à l'émergence d'autres. C'est pour cette raison particulière que les ethnographes soviétiques accordent une attention considérable à la question 
des origines des peuples, ou ethnogenèse. » $(1980: 160)$

La théorie des ethnies, dans l'ethnografya soviétique, rencontre une difficulté majeure à laquelle sont confrontés tous les raisonnements de type évolutionniste : d'un côté, pour saisir le changement dans les collectifs humains, il convient d'abord de les catégoriser, c'est-à-dire de les fixer dans une classification "à plat ", nécessairement inscrite sur un plan synchronique, qu'impose cette démarche méthodologique, même si la finalité reste de restituer les ethnos à l'histoire, et de les déplacer vers un plan diachonique. Dans ce sens, l'ethnographie soviétique a procédé à une essentialisation des ethnos, en désignant des traits distinctifs identifiables (langue, coutumes, croyances...) et un sentiment d'appartenance sociale bénéficiant d'une certaine stabilité (Bromley, 1978 : 17), alors que parallèlement - et encore une fois paradoxalement - l'étude de leur évolution suppose de placer au cœur de l'analyse la question des changements, c'est-à-dire, de leur instabilité. Ce qui intéresse donc en fait l'ethnographie soviétique à travers le concept d'ethnogenèse, c'est moins les processus de métamorphose que ceux de formation ethnique, ou d'ethnies, comme formes constituantes pour des peuples dans l'Histoire, avec une identité à la fois épistémique (que la science forge ou confirme) et sociocritique (que les sujets individuels ou acteurs collectifs ressentent ou revendiquent).

Les récentes théories de Lev Gumilev, qui placent l'ethnogenèse au cœur de l'analyse, reposent encore sur une analyse historique de type évolutif : les ethnoi (ethnos) se développent par des périodes identifiées, et possèdent parallèlement un niveau de puissance qui se mesure à travers le concept étrange de passionarity, difficilement traduisible. La théorie ethnogénétique de Gumilev se présente comme une description des cycles de formation et de transformation des groupes humains, qui suit un processus en stades : émergence, développement, point culminant, inertie, régression, commémoration. Le niveau le plus élevé de ce cycle correspond au plus haut degré de passionarity, ce qui est l'une des causes, selon Gumilev, des conflits entre ethnos. Cette conception évolutionniste, naturaliste (même si l'auteur s'en défend) et cyclique n'est pas sans rappeler les temps révolus d'une anthropologie (notamment diffusionniste) qui pensait les peuples comme des Geist, ou les civilisations comme soumises à des cycles d'essor et de déclin. Le projet de Gumilev est pourtant celui d'une " ethnologie » qui se donne comme objet les ethnoi dans une histoire universelle qui est celle de leurs relations, de leurs transformations et - pour certains - de leur disparition. "History is a single process » affirme-t-il, dans son ouvrage Ethnogenesis and Biosphere on Earth (1994). La définition de l'ethnos est des plus délicates : il n'est pas " société ", ni « nation », ni « culture ", ni même " ethnie », même si son existence repose sur des processus propre à la première, et qu'il s'étend dans des espaces à l'image de la deuxième, qu'il emprunte nombre de ses formes à la troisième et qu'il contribue à distinguer les groupes humains les uns des autres, à l'instar de la quatrième.

Si les thèses de Gumilev trouvent encore des partisans de l'autre côté de la Volga, elles ont reçu peu d'écho en Europe occidentale, où, lorsqu'elles sont diffusées, elles font parallèlement l'objet de violentes 
critiques, portant notamment sur la réification de l'ethnos, le caractère évolutionniste des modèles de l'histoire et l'ethnicisation à outrance des différences sociales et inter-sociétales, comme énoncées par Thornike Gordadze (2001). La notion d'ethnogenèse a donc des racines soviétiques, et s'inscrit dans un espace de débat qui touche à l'identité historique et aux transformations des groupes et des sociétés de ce que l'on qualifiait il y a encore peu de "bloc de l'Est ». L'ethnographie soviétique s'est en effet d'abord, et surtout, intéressée à ces " autres intérieurs » que furent, et sont encore, les « minorités nationales ». Comparative sur le plan théorique (en soumettant, par exemple, la consistance des ethnos soviétiques à celles d'autres " ethnos " occidentaux, comme l'ethnos "français ", Bromley, 1978), elle reste néanmoins circonscrite à des explorations empiriques restreintes à un espace national, et a donc peu développé la réflexion post-coloniale dans laquelle se sont engagés les traditions anthropologiques d'Amérique du Nord et d'Europe occidentale. Le cas est aussi vrai pour l'anthropologie chinoise, dont Wang Mingming a récemment dressé un panorama historique : comme l'ethnographie soviétique, l'ethnologie chinoise s'est longtemps consacrée à l'étude des " minorités nationales » (shaoshu minzu), sous l'impulsion des orientations politiques du gouvernement de Beijing, contribuant ainsi à réifier des ethnies différentes, porteuses d'une altérité assignée par les instances académiques et politiques centralisées (Mingming, 2002). L'usage de cette notion "à l'Ouest " ne relève donc que peu ou pas d'un prolongement de ces réflexions, à plus forte raison lorsqu'elles concernent des pays à dominante capitaliste industrielle (qu'ils en soit les inspirateurs, comme les pays d'Occident, ou les destinataires, comme les pays d'Asie et d'Afrique colonisés). Il s'agit donc moins d'un retour que d'une réinvention du concept d'ethnogenèse dans les Globalization Studies, et l'œuvre qui a cristallisé un tel déplacement est sans aucun doute l'Ethnogenesis d'Eugen Roosens (1989).

\section{Orientations et objet des Globalization Studies}

Les Globalization Studies, de fondation récente, représentent un domaine d'étude aux contours flous, et à l'objet imprécis. Il s'agit, certes, d'un champ interdisciplinaire tourné vers l'étude de la mondialisation, mais admettant une grande variété de disciplines, terrains, perspectives et épistémologies qui le composent. En outre la continuelle fragilité de l'objet (pour lequel n'existe actuellement aucun consensus de définition, sauf à le traiter de manière nominale comme une réalité historique, GEMDEV, 1999) confère aux Globalization Studies l'aspect d'une mosaïque plutôt que celui d'une discipline fermement établie sur des fondations conceptuelles stables et unifiées. En revanche, ce sont de mêmes questions fondamentales qui sont posées, examinées et discutées : l'origine, l'extension, les formes et les impacts de la mondialisation, pour simplifier à l'extrême. La tripartition usuellement tracée entre "mondialité » (un état), « mondialisme » (une idéologie) et « mondialisation» (un processus) 
permet de conférer à ce dernier terme la dimension dynamique et diachronique qui le caractérise. La mondialisation est ainsi définie comme relevant du mouvement de l'histoire ou de l'histoire en mouvement (mais une histoire, dans les deux cas, universelle) et comme moteur du changement ou des métamorphoses des sociétés humaines, sur tous les plans - du macroscopique, les "structures " politiques ou économiques étendues à l'échelle d'une nation ou de plusieurs, au microscopique, en pénétrant et marquant de son emprunte les espaces privés et ordinaires (par la télévision, la consommation, la culture matérielle, etc.).

Pourquoi l'ethnicité ressurgit-elle à la fois sur la scène sociale et intellectuelle, parallèlement à la montée en puissance des théories de la mondialisation? Plusieurs versions se partagent actuellement l'espace des débats sur la mondialisation. Une première, que l'on qualifiera d'optimiste, dépeint un monde " ouvert ", fait de mélanges, de contacts et d'interactions culturelles, qui est le théâtre d'emprunts et de métissages à l'origine d'une sorte de transculturation du monde, notamment dans le domaine religieux (Luca \& Burrell, 1999). Une seconde, nettement plus pessimiste, représente au contraire le monde actuel comme un espace de forces antagonistes, caractérisé par la conflictualité et la tension entre les groupes humains. C'est donc moins une interpénétration culturelle entre des groupes pacifiques, qu'une confrontation généralisée des sociétés sur le mode de la concurrence, de la confrontation et du conflit. Et c'est précisément cette conflictualité qui suscite la montée en puissance des revendications identitaires (Friedman, 1988; Dunaway 2003). Les ferments théoriques de l'ethnogenèse se trouvent donc dans l'essor rapide des thématiques de l'«identité » dont les sciences sociales se sont massivement (ou ont été) saisies : partout, ce sont des identités " en mouvement », " hybrides ", " en mutation ", " renégociées ", "transformées »... dans le contexte de la mondialisation. C'est donc ce dernier (ou l'interprétation qui en est faite) qu'il convient maintenant d'interroger.

\section{EthNiCité et MONDIALISATION}

L'usage commun veut que le concept d'ethnogenèse se traduise par : " le processus par lequel un groupe est considéré ou se considère luimême, sur le plan ethnique, dans un paysage social plus large qui est celui de son émergence ». C'est donc un phénomène diachronique, parce que processuel, de singularisation d'un groupe humain par rapport à un autre. Or, la notion d'ethnogenèse, si elle s'impose assez rapidement, mais de manière dispersée (non paradigmatique), dans les Globalization Studies, y apparaît comme un opérateur théorique aux significations et aux usages quelque peu différents.

L'extension des réflexions et l'accroissement des occurrences du concept d'ethnogenèse sont de précieux outils de lecture des visions sous-jacentes de la mondialisation. Certes, le terme ne possède pas la surface lexicale d'autres " mots valises » des sciences de l'homme, comme « diaspora » ou « mondialisation » (Guillen, 2000) qui ont colonisé 
ce vocabulaire. Mais il s'invite de plus en plus régulièrement dans les débats sur les transformations du monde, les réinventions de l'Histoire et des identités collectives.

À l'évidence, c'est la version différentialiste qui s'impose, qu'elle soit par ailleurs issue de matrices diffusionniste (sur l'idée de " mélanges généralisés " des populations et des cultures) ou structurofonctionnaliste (c'est alors le système-monde, lui-même unifié, qui suscite des expressions locales de la différence). Dans tous les cas, comme le signalent Preyer \& Bös, la mondialisation crée de nouvelles frontières, des zones " entre-deux ", des territoires interstitiels qui sont les nouveaux lieux de production de l'ethnicité. Et c'est dans ce contexte que ressurgissent conjointement les questions d'identité, d'ethnicité et d'ethnogenèse. L'idée, momentanément énoncée, d'un possible nivellement des différences culturelles, sous le poids d'une " modernité ", d'abord, d'une "mondialisation ", ensuite, (avènement d'une société mondiale uniformisée sur le modèle des nations industrielles telles que l'Occident les a vu se développer) avait prématurément conduit à diagnostiquer une homogénéisation du monde, via celle des sociétés.

Le psychosociologue Young Yun Kim, qui a récemment consacré une étude à la dynamique de production de l'identité ethnique et de ses contextes à l'échelle des individus, introduit l'article en question ("From Ethnic to Interethnic ») par une courte formule qui sonne comme un slogan : "We live in a era of clashing ethnic identities " (2006 : 283), avant de questionner les conditions, modalités et conséquences de la production d'identités ethniques dans un monde au " paysage fracturé » où la " "diversité" et le "multiculturalisme", adoptées par nombre de sociétés, est à tout le moins un point de tension et de contention" (2006 : 284). Que l'ethnicité ait gagné en surface sémantique ne surprend finalement pas, car c'est l'ensemble d'un paradigme - celui du mondialisme - qui s'exprime à travers ses récurrences lexicales.

L'idée générale qui se dégage d'un certain nombre de travaux saillants sur la question (notamment ceux de Jonathan Friedman, 1988) est que la mondialisation (globalization), quelle que soit par ailleurs la forme qu'on lui assigne (world-system à la Wallerstein ou monde en flux à la Appadurai, 1998, 2001), n'a évidemment dissout ni les cultures nationales ou locales, ni les expressions d'une singularité ethnique. Les limites du modèle de l'homogénéisation culturelle se font ainsi saillantes face à la théorie alternative, et de plus en plus consensuelle, de la différenciation culturelle. Ce regain d'intérêt pour les questions d'identité et d'ethnicité trahit en effet les présupposés des théories dominantes de la modernité et de la mondialisation. C'est parce que le " grand récit » (pour ne pas dire «mythe ») de la modernité présente cette dernière comme déstructuration, mise en mouvement et recomposition permanente des identités (individuelles ou collectives, sociales ou ontologiques) qu'elle offre une lecture de la vie sociale dans les mondes contemporains : l'image d'un monde fluide, instable (conforme à la figuration très expressive de "modernité liquide » - liquid modernity - énoncée par Zygmunt Bauman) justifie la multiplication exponentielle des recherches sur la question identitaire (Bauman, 2001). Mais les conditions de la modernité sont-elles celles 
de la mondialisation? Des auteurs comme Anthony Giddens tendent à confondre les deux concepts - ou du moins à les associer dans une même analyse (1994). D'autres, comme Stuart Hall, distinguent au contraire les deux concepts : la modernité, dissolvante pour les identités, la mondialisation, qui agit à l'inverse comme un catalyseur identitaire (1991).

Selon Jonathan Friedman, la mondialisation est à l'origine d'une « renaissance de l'ethnicité » $(1988$ :453). Il résume ainsi une posture intellectuelle qui est largement partagée au sein des Globalization Studies. Bien ancrée dans la perspective des Cultural Studies, la posture de Stuart Hall, évoquant de "nouvelles et anciennes identités et ethnicités " (old and new identities, old and new ethnicities, in King et al., 1991), suggère que le contexte contemporain est celui de la « re-création, de la reconstruction de l'imaginaire, des places connues et connaissables, face au global postmoderne qui a détruit les identités de lieux spécifiques, les a absorbées dans le flux postmoderne de la diversité " (The local and the global, in King et al., 1991 :35). Dans le même ordre d'idées, John Tomlinson s'oppose à l'idée que la mondialisation détruit les identités, et estime au contraire qu'elles restent un «trésor » (treasure) pour les individus et les groupes, susceptibles d'être utilisées comme un " pouvoir culturel " (cultural power), et qu'elles prolifèrent, en ce sens que la modernité offre précisément les conditions propices à l'institutionnalisation de ces identités reformulées au prisme de nouvelles formes de culture et de vie sociale, légitimées par la "logique interne » de la modernité (Tomlinson, 1999 : 269-277). Tomlinson rejoint ainsi indirectement la posture d'un Martin Albrow qui, dans son œuvre majeure mais controversée, The Global Age, consacre une section particulière (mais relativement courte) à la question, «Ethnicity in the Global Age » (1997 : 197-200). L'ethnicité (locale) y apparaît comme le fruit actuel (et non pas la résurgence traditionnelle) des identités, travaillées par les forces globales. L'accent politique mis par Albrow sur l'ethnicité contemporaine n'a rien de vraiment original, mais l'auteur rappelle avec raison que l'ethnicité est liée au politique, soit parce qu'elle est modulée par des conditions particulières, soit parce qu'elle fait l'objet de politiques ou de stratégies (à l'image du concept anglais de politics). Encore faut-il que ces liens soient précisément éclairés, ce à quoi cet article s'appliquera ultérieurement.

\section{L'ETHNICITÉ COMME RÉPONSE À UNE II CRISE ॥}

Dans l'article introductif d'un numéro spécial de l'International Journal of Comparative Sociology, le sociologue Edward A. Tiryakian défend l'idée que les formes de l'ethnicité contemporaines sont la conséquence des crises politiques associées à la modernité, en l'occurrence les crises des États-Nations. Pour Helmuth Berking, l'affaire est entendue : Ethnicity is Everywhere (2003, rééd. 2004). La synthèse éclairante que le sociologue allemand fait de la montée en puissance thématique, dans les débats sociaux et dans les milieux académiques de la catégorie d' " ethnique ", est selon lui très directement liée à la mondialisation : les flux 
d'idées, les mélanges culturels, les dynamiques sociales entre collectifs humains toujours plus proches les uns des autres... soumettent précisément les identités individuelles et collectives, auparavant vues comme relativement "stables ", à un impératif de reformulation. Mais sous la plume de Berking, ce ne sont pas tant les processus et conditions factuelles de la dite mondialisation, que la dialectique de l'universalisation et de la particularisation, inhérente au procès de mondialisation (qui en est donc un produit associé mais autonome), qui engendrent une politique généralisée de la revendication identitaire, de fabrique des différences, et qui contribue à la construction de «fictions totalisantes » des cultures et des groupes ethniques, d'autant plus essentialisées qu'elles offrent l'une des rares possibilités de créer des "îlots d'identité » dans une " mer de contingence » (2004 : 59). Ce qui explique dans un autre registre que celui de la modernité, l'ethnicisation actuelle des identités culturelles oppositionnelles, discriminantes - fondées sur une représentation de soi, des groupes d'appartenance, de la " communauté imaginée " (pour filer la métaphore très usitée que Benedict Anderson applique au nationalisme), sur un plan synchronique, celui d'appartenance symbolique ou sociale hic et nunc, et celui, diachronique, de la référence à un passé fondateur, souvent mythifié et toujours reconstruit dans le cadre d'enjeux sociopolitiques contemporains. Dans ce sens, la mondialisation ne liquéfie ni ne dissout les identités collectives : au contraire, elle les réifie, les cristallise. Elle produit donc d'un côté de l'hybride, de l'hétérogène sous l'impulsion des forces du métissage, de l'autre du pur, de l'homogène, en réponse aux craintes que suscitent les métamorphoses rapides des repères culturels et des cadres sociaux.

L'ethnicisation des identités participe donc d'une violence de la mondialisation et par la mondialisation faite aux identités collectives. Mais cette violence n'est pas que celle des mots et des idéologies. Selon Wilma A. Dunaway, les rapports entre mondialisation et ethnicité se comprennent ainsi à la lumière de la conflictualité des rapports sociaux et sociétaux, et des lignes de tension entre les forces globales et locales : la mondialisation est ainsi à l'origine d'un processus de «fragmentation ethnique » $(2003: 4)$ qu'illustre la diversité actuelle des revendications identitaires singulières. Pour autant, et contrairement à l'image que l'on pourrait s'en faire, à mesure que la mondialisation avance, il n'existe pas de preuves statistiques de son impact sur les conflits ethniques : au contraire, depuis un siècle, on observe une incidence plus faible $d u$ " paramètre ethnique » dans les affrontements et violences intersociétales ou intergroupales (Dunaway, 2003 :5). Il est surtout important de rappeler que ces « mesures » de la conflictualité contemporaine font apparaître que la violence est moins inter-étatique qu'intra-étatique (Martin, 2005), et donc qu'elle touche le plus souvent au problème des rapports entre groupes dominants et « minorités » ou groupes " ethniques ", qu'à celui des rapports entre nations. C'est ce que montrent par exemple les travaux de Guillaume Boccara : la tension permanente entre l'État et les indiens Mapuche au Chili a occasionné des résistances et une volonté à l'auto-détermination qui s'est traduite par l'affirmation d'une singularité ethnique d'autant plus complexe qu'elle a perpétuellement changé de labels identitaires (1999). 
Le propos de Dunaway fait écho à un débat interne aux Globalization Studies qui peut se lire ainsi : la violence, les conflits, discriminations, expressions du racisme et les ségrégations en tous genres sont-ils des effets de la mondialisation, des résistances à celle-ci, donc des réactions (c'est notamment l'idée défendue par I. Wallerstein) ou, au contraire, sont-ils des paramètres propres au développement d'un système-monde (selon Friedman)? Pour Wilma Dunaway, la conflictualité ethnique contemporaine est fonction des tensions entre, d'un côté, les forces de «l'hégémonie culturelle » (celles qui poussent à l'homogénéisation de certains compartiments des sociétés inscrites dans la mondialisation) et d'un autre côté, celles de "l'hétérogénéité ethnique ", c'est-à-dire, la tension entre d'un côté, les efforts d'intégration des groupes et des nations dans un système-monde (celui du capitalisme globalisé), et, de l'autre, les résistances de ces derniers au projet de civilisation qui accompagne l'extension de ce même système global (2003 : 7). Mais le processus d'ethnogenèse n'est, dans ce contexte pas qu'une réaction locale à l'avancée du capitalisme mondialisé : Dunaway rappelle en effet la construction historique des « minorités ethniques » par les pouvoirs coloniaux (un peu partout sur la planète) - minorités qui, par leur existence, légitimaient la domination coloniale. La création de nouvelles identités ethniques par « mobilisation du passé et des ressources culturelles " n'est rien d'autre que la quête de souveraineté de ces anciennes " minorités indigènes" (2003 :7-9). Ainsi, les nouvelles ethnicités produites dans l'histoire, ou ethnogenèse, et puisant dans l'histoire admettent-elles une certaine continuité (au moins dans leur processus) avec une période antérieure à la mondialisation (si on décide d'adopter pour celle-ci une chronologie courte, celle de la mondialisation du capitalisme, remontant au XIX ${ }^{\mathrm{e}}$ siècle).

\section{EthNOGENÈSES ET CONDITIONS POLITIQUES}

La convocation d'un terme aussi peu mobilisé dans les sciences de l'homme occidentales que celui d'ethnogenèse n'est pas la garantie d'une unité de sens. Sur sa propre échelle - celle de ses usages - il convient alors de procéder à une révision de ses significations et de sa portée. À travers un premier repérage des usages, il est possible de distinguer plusieurs significations assignées à la notion d'ethnogénèse. Elle est d'abord un processus objectif de création de nouvelles identités. Dans un Working Paper de Pioneer (Pioneers of Europe's Integration from Below: Mobility and the Emergence of European Identity amonh National and Foreign Citizens in the $E U$ ) daté de juillet 2003 (et disponible sur 1'Internet) Ettore Recchi et Tina Nebe parlent simplement d'ethnogenesis comme d'un processus d'élaboration de nouvelles identités culturelles. Selon Miguel Alberto Bartolomé (2004), l'ethnogenèse admet en fait deux acceptions : elle est soit le processus historique par lequel se configurent les identités collectives, soit le processus d'émergence de nouvelles communautés qui se désignent comme " ethniques ». Elle n'est donc pas à proprement parler une redynamisation ethnique, mais un processus d'actualisation 
identitaire de groupes dont la position dans des configurations politiques larges (ensembles nationaux) ou les structures politiques avaient limité les chances de mobilisation sociale et de revendication identitaire.

La littérature offre des formulations combinatoires entre les deux modèles, celui de la renaissance " objective " ou de l'invention " instrumentale » d'identités collectives dans un contexte marqué par le politique. Dans une livraison déjà ancienne de la revue Ethnicities, Frank F. Montalvo et G. Edward Codina (2001) posent que l'ethnogenèse est le processus objectif par lequel se crée l'identité (métissée) des Mexicains, mais à partir duquel se mesurent les intrumentalisations contemporaines de leur identité. Montalvo et Codina s'intéressent alors aux manipulations de la notion identificatoire de " race » et des phénotypes associés pour la définition de l'identité des métis (mestizos) qui procèdent à des divisions là où les catégories identificatoires qui leur sont assignées tendent vers une forme d'homogénéité. On voit donc ici globalement que l'identité, l'ethnicité, et l'ethnogenèse peuvent être articulées différemment (comme des " composantes » ou de "moments " les unes des autres), et surtout articulées à des cadres théoriques peu éloignés, mais admettant certaines différences marquées.

Le grand théoricien actuel de l'ethnogenèse reste évidement Eugen Roosens, qui lui a consacré un ouvrage en 1989 : Creating Ethnicity. The Process of Ethnogenesis. Le théoricien émergent de l'ethnogenèse décrit ces processus de création de nouvelles " ethnicités " ou de "nouvelles cultures » (l'ethnicité étant fondée sur des référents culturels) comme se faisant précisément en opposition aux catégories imposées par voie politique : les Chicanos, qu'il a étudiés, ne sont par exemple ni " mexicans ", ni « americans », ni « mexican-americans ». Dans ce sens, l'ethnicité est un construit relationnel, éclairant les aspects dynamiques et situationnels de l'ethnogenèse, qui n'est rien d'autre que le processus global de création de nouvelles ethnicités. Mais l'analyse de Roosens est tributaire d'un cadre théorique et d'une configuration socioculturelle spécifique : les réponses post-coloniales d'un groupe particulier à des sous-catégories identitaires (hyphenated) par la création ou la valorisation de catégories propres, un phénomène que d'aucuns, par comparaison, considèrent comme un mécanisme récurrent de l'ethnicité et, partant, de l'ethnogenèse, transposable à d'autres groupes (Flannery, Reise \& Yu, 2001). Il est étrange, sur cette base, que Roosens soit accusé d'essentialisme (comme le fait Yun Kim, 2006 :286) vu le caractère interactionniste et dynamique du modèle qu'il propose.

\section{VARIATIONS ETHNOGÉNÉtIQUES :}

\section{INVENTIONS, RÉINVENTIONS, RÉÉCRITURES DE L'HISTOIRE}

Par delà ces stratégies à l'œuvre au niveau des sujets qui la mobilisent, l'ethnogenèse apparaît, dans son contenu, comme relevant d'une réinvention politique de l'histoire. Avec Jones et Hill-Burnett cités par 
George Morgan dans son étude sur les revendications à l'autochtonie des aborigènes d'Australie (2003 :442), l'ethnogenèse apparaît comme un processus politique qui permet de fonder une identité à partir de l'image d'une communauté archaïque (imaginée). Dans ce sens, elle est revitalisation de formes culturelles ou sociales antérieures (elle possède une dimension diachronique), mais procède d'une réinvention. Parce que la formulation d'une identité « ethnique » passe nécessairement par référence à un passé, quel que soit par ailleurs son exactitude historique, comme l'a montré Max Weber, les discours identitaires contemporains sur l'identité (mais on peut le supposer à l'identique pour les discours « anciens ») sont de nature peu ou prou de nature « ethnogénétique »: ils construisent un passé sur lequel se fondent et se légitiment les identités actuelles.

Que cette ethnogenèse à finalité politique participe d'une réactivation ou d'une invention du passé ouvre un débat épineux. Toujours est-il qu'elle trouve écho dans les instances les plus légitimes de débat sur ces questions. José Bengoa, membre du groupe de travail sur les minorités à la Commission des Droits de l'Homme qualifie, dans un rapport intitulé Minorités et autodétermination (daté du 23 décembre 2003), l'ethnogenèse comme processus de « renaissance culturelle » pour des minorités auparavant dominées, ratifiant ainsi une conception postcoloniale désormais largement répandue dans les sciences de l'homme. Derrière l'idée d'une " renaissance » des formes culturelles point néanmoins un certain essentialisme, comme si les cultures des minorités se revivifiaient en l'état après avoir subi la pression coloniale. Les travaux de George Morgan (2003) dévoilent en outre toute la complexité de la question : il montre que l'identité aborigène (Aboriginality), réaction urbaine à la colonisation s'exprimant à la manière d'un Black Power, est considérée comme procédant d'une « redécouverte" plus que d'une réinvention des traditions mobilisées dans cette affirmation identitaire. Jones et HillBurnett (cités par Morgan, 2003 :440) inscrivent, sur cette base, le mouvement pan-aborigène dans un processus ethnogénétique. Mais il n'est pas lui-même une ethnogenèse.

\section{L'ETHNOGENÈSE COMME INSTRUMENT POLITIQUE}

C'est ainsi l'horizon conceptuel d'une anthropologie politique qui a permis de rompre avec les conceptions objectivistes de l'ethnogenèse, qui pourtant se maintiennent dans les registres d'analyse précédemment évoqués. Le concept d'ethnogenèse s'avère alors relever de l'instrumentalisme politique - et non pas d'une simple épistémologie instrumentaliste à la Barth. Les travaux de Pietro Scarduelli (2005) sur l'émergence récente, en Italie du Nord, d'un mouvement politique d'extrême-droite et sécessionniste, (la Ligue du Nord) contribuent à élargir ce champ d'application de la notion d'ethnogenèse. Scarduelli qualifie l'ethnogenèse de "stratégie politique " : sans entrer dans de longues considérations sur la définition même de l'ethnogenèse, l'auteur décrit les modalités 
de construction d'une identité régionale et d'un territoire particuliers (la Padanie), à travers l'invention d'une histoire commune, de héros, de saints, de guerres, d'une mythologie, pour susciter un sentiment d' "âme » nationale et nationaliste - calquée sur le pan-celtisme à l'œuvre dans d'autres régions d'Europe. Le cas de la Padanie montre que la création d'une ethnicité distincte ne peut se passer d'une création de tradition, c'est-à-dire, d'une réécriture instrumentalisée du passé, ce à quoi s'affairent les diverses mouvances nationalistes en Europe. Dans les débats sur les rapports entre ethnicité et histoire - l'identité ethnique est soit un produit de l'histoire, ou d'une histoire, soit elle est historicisée mais aussi historicisante, elle fait l'histoire ou une histoire -, Scarduelli choisit une posture intégrative qui montre la validité conjointe des deux approches (2005 : 148). Mais ces travaux, et les réflexions qu'ils suscitent, invitent donc à questionner les rapports entre mondialisation et ethnogenèse. À moins de voir partout se manifester les effets de la mondialisation, on ne peut pas vraiment affirmer que l'ethnogenèse padanienne lui soit directement liée. À tout le moins, elle aurait également pu être éclairée sous l'angle de la "modernité ", et dans le cadre - devenu désormais classique - des inventions de traditions depuis la publication du célèbre livre éponyme de Hobsbawn et Rangers (1983 pour la version anglaise, récemment traduit en France).

Il n'est pourtant nul besoin que le nationalisme se fonde sur des enjeux politiques aussi marqués, et que le processus d'ethnogenèse qui lui est associé participe de ces velléités séparatistes pour qu'il œuvre à la recomposition et à la "transistorisation » du passé. Martha van der Bly a par exemple tout récemment (2007) montré comment, dans le petit village de Leixlip, en Irlande, c'est l'implantation d'une filiale d'Intel (le " géant » informatique), qui a amené la ville à se réinventer une histoire, des ancêtres, un mythe national, des héros... c'est-à-dire, à produire une ethnogenèse, certes patriotique, mais pas nationaliste. Ici, les liens entre ethnogenèse et mondialisation sont tout à fait patents, comme ils le sont par ailleurs dans la réinvention de l'« authenticité culturelle » des Aborigènes d'Australie, confrontés à l'évolution rapide des textes de lois favorisant l'expression de leurs droits et de leur revendication à la singularité " ethnique ", impulsée par le mouvement transnational de l'autochtonie (Duelke, 2005).

Ainsi, au regard des différentes manières d'écrire l'histoire des collectifs humains, l'un des points de distinction majeurs entre l'évolutionnisme de l'ethnografya soviétique et les théories du changement social et identitaire qui prévalent dans les Globalization Studies réside dans le rôle que chacune des matrices théoriques assigne aux sujets collectifs dans leur ethnogenèse, et, partant, dans l'ontologie qu'elles confèrent à ce processus : autant l'ethnographie soviétique considère que la formation des ethnos est fondée sur des processus « naturels et historiques » et, en conséquence, ne saurait être attribuée à une quelconque volonté collective (Bromley, 1978 : 16), autant l'écrasante majorité des usages actuels de la notion d'ethnogenèse dans les Globalization Studies signalent au contraire que le processus n'est non seulement pas exactement celui de l'histoire « objective » d'un précipité ethnique particulier, mais une réinvention de 
l'histoire, et qu'en outre, celle-ci procède largement de stratégies largement plus intentionnelles que la théorie des ethnos de l'avait envisagé.

La résurgence du concept d'ethnogenèse suppose ainsi de nouvelles définitions et de nouveaux contextes d'usage. Tout concept est l'objet d'une permanente redéfinition. Cette courte cartographie avait vocation à l'illustrer. Mais les questions d'ethnicité et d'ethnogenèse n'interrogent pas que le monde : elles interrogent en miroir les manières dont l'académie voit et théorise le monde, et, depuis Robertson pour la sociologie, ou Appadurai pour l'anthropologie, le champ d'analyse qui s'intéresse aux effets de la mondialisation de la sociologie ou de l'anthropologie sur la sociologie ou l'anthropologie de la mondialisation est ouvert. On pourrait très bien s'arrêter à l'examen de ces rapports réciproques entre les concepts ou modèles théoriques, et les conditions de leur production : l'épistémologie contemporaine s'en trouverait pleinement satisfaite. Il est néanmoins possible d'aller un peu plus loin, pour questionner moins les concepts euxmêmes que les contextes dans lesquels ils sont formulés, en l'occurrence, les idéologies de la modernité et de la mondialisation. J'adresserai ici un certain nombre de remarques critiques à ces deux cadres de pensée.

\section{US Et ABUS DE L'II ETHNIQUE II, PORTÉE ET LIMITES DU MONDIALISME}

L'inflation autour de la catégorie d'« ethnique » et ses variantes (ethnicité, ethnicisme, ethnicisation, et donc ethnogenèse...) procède en premier lieu d'une contagion excessive de l'ethnique à l'ensemble des phénomènes identitaires. Certes, la notion d'ethnique est suffisamment étendue pour recevoir les interprétations les plus variées - ce qui en fait d'ailleurs l'un des points faibles. Mais dès lors que s'expriment des revendications collectives, socle potentiel des particularismes, communautarismes et nationalismes, n'est-on pas tenté de les regrouper un peu hâtivement sous la catégorie de l'« ethnique »? Si la tendance à qualifier d'« ethnique » toute forme d'identification collective dans les sociétés "pré-modernes » qui prévalait dans certains travaux a fait son temps, la récurrence contemporaine des expressions d'une singularité inscrit le « renouveau ethnique » au cœur de la modernité (Schnapper, 2005). Ce désenclavement de l'« ethnique» de l'archaïsme ne fait toutefois pas consensus. Tout n'est pas " ethnique ", dès lors que le regard se porte sur les éléments qui confèrent leur singularité aux groupes sociaux - et la manière dont ils les mobilisent ou pas. C'est ainsi qu'au Brésil, pays qui, par excellence, a suscité force débats sur la diversité culturelle et les stigmates sociaux qui l'affectent, Livio Sansone a démontré que l'identité « noire » (Blackness) brésilienne n'est pas raciale mais culturelle, tout en étant affranchie de toute ethnicité (de tout investissement symbolique et de mobilisation politique autour de l'identité) - d'où le titre de son livre : Blackness without Ethnicity (2003).

La critique peut - et doit - être étendue aux paradigmes théoriques qui ont porté l'ethnicité et l'ethnogenèse au premier plan des questions actuelles sur l'identité : encore une fois, la modernité et la mondialisa- 
tion. L'une et l'autre ont en effet vocation à proposer une nouvelle conception de l'histoire : pour rependre les termes de Jan Aant Scholte, on assisterait, avec la mondialisation, à " la fin des modèles endogènes de l'histoire " et à une tentative de les réintégrer dans une perspective globale (1993, cité par Spybey, 1996 : 157). Ce que le postmodernisme avait introduit comme variété, au péril de la dispersion et de l'abdication de la pensée, (au principe que tout se vaut et réciproquement), se trouve réaligné par le mondialisme dans un modèle unifié. C'est donc moins la fin de l'histoire (pour emprunter, mais dans une conception différente, la célèbre expression à Francis Fukuyama qui l'avait réservée à une histoire socio-économique du monde) que la fin des histoires, sacrifiées sur l'autel de l'œcumène global dont la puissance explicative ne me semble pas seulement résider dans sa portée théorique, mais aussi dans son inscription dans le pouvoir de l'académie - même si celle-ci n'est plus à proprement parler « occidentale ». En retournant le propos sur le mode anthropologique, c'est-à-dire en considérant leurs conditions de formulation mais aussi (et surtout) le sens que les groupes leur confèrent, s'il existe des ethnogenèses différentes, ce sont au contraire autant de nouvelles versions des histoires culturelles ou des versions historicisées des identités collectives qui contredisent l'idée de prédominance d'une histoire mondiale unifiée (qui est en fait une histoire de la mondialisation, voire de l'idéologie mondialiste).

C'est en second lieu la question de la nouveauté des phénomènes passés au prisme du modernisme et du mondialisme qui se pose avec acuité. Parallèlement à Tiryakian, Janet Abu-Lughod (1991, citée par Tiryakian, 2004) s'inscrit dans ce mouvement de pensée qui affirme l'existence d'une différence radicale entre les conditions de la mondialisation ou de la modernité, et celles dans lesquelles vivaient les sociétés traditionnelles. Les mélanges, les transformations des cultures, et conséquemment, des identités, sont des processus récents et plus caractéristiques des sociétés modernes que des sociétés traditionnelles vivant, selon l'expression de l'historien français Pierre Chaunu (cité par Spybey, 1996 : 157), dans des « univers cloisonnés ». Cette représentation d'un monde prémoderne constitué d'une constellation d'isolats culturels qui ne se seraient « connectés » que tardivement dans l'histoire, "découvrant " par là-même leurs identités respectives, n'avait pourtant pas résisté à l'implacable démonstration de son indigence faite par Lévi-Strauss dans Race et Histoire (1955). Et Steve Fenton, un spécialiste des ethnic studies relativise encore le propos en mettant en question les rapports entre ethnicité et modernité, et le rapport mécanique qui les associe sous l'angle de causes ou de conséquences forcément mutuelles (2004). Le problème des versions à coloration nationaliste ou fondamentaliste de l'ethnicité, c'est qu'elles sont généralement interprétées comme des réactions face à la mondialisation (comme aliénation par le système-monde, ou par l'expansion qualifiée d'impérialiste des modèles occidentaux. Mais la réaction de qui? De mondes et de sociétés « pré modernes » ou périphériques à la mondialisation, et cette figuration des sociétés autres, réactives et résistantes, ne manque pas de soulever des questions essentielles. Ce qui ramène évidemment au cœur du débat sur les rapports entre identité, 
ethnicité et mondialisation. La réémergence récente du concept d'ethnogenèse est corrélatif d'un questionnement global sur les identités culturelles, sous deux versions opposées de la mondialisation : l'une, destructrice, l'autre, source de créativité. Il n'est pas ici question de reprendre les termes d'une discussion longuement débattue, mais d'en examiner les soubassements idéologiques au prisme d'une critique anthropologique. C'est précisément cette tendance à projeter, paradoxalement, des formes résiduelles de primitivisme (géographique ou historique) sur un phénomène qualifié de moderne ou considéré comme tel par sa pleine inscription dans la mondialisation, qu'il faut ici dénoncer avec virulence. Ce qu'énonce avec humour John Tomlinson, lorsqu'il entreprend de rendre le mythe fondateur du mondialisme sous une forme narrative :

"Il était une fois, avant l'ère de la mondialisation, un temps où il existait, entre le lieu géographique et l'expérience culturelle, des connections locales, autonomes, distinctes, bien définies, robustes et culturellement préservées. Ces connections constituaient l'identité culturelle d'une personne et d'une communauté. Cette identité était quelque chose que les gens "avaient" simplement, dans le cadre d'une possession existentielle non problématique, un héritage, une continuité avec le passé. L'identité était "une sorte de trésor collectif pour les populations locales", mais quelque chose de fragile qu'il fallait préserver. »

Le récit de cet Âge d'or originel se poursuit par l'introduction des forces du chaos : "Car dans ce monde, elles furent brusquement secouées (apparemment dans les années 1980). Par le pouvoir corrosif de la globalisation. La globalisation, comme le dit l'histoire, s'est répandue comme un torrent à travers les cultures du monde, détruisant, sur son passage, les localités stables, déplaçant les gens, entraînant une homogénéisation de l'expérience culturelle portée par le marché, et oblitérant les différences entre les cultures localement définies qui constituent nos identités. D'où l'idée que la globalisation est un processus général de déperdition de la diversité culturelle [...].»

Mais ce mythe, comme bien d'autres, peut se voir substituer à un autre : «Une autre histoire peut être racontée, conclut Tomlinson, la globalisation comme l'une des forces les plus significatives de création et de prolifération des identités culturelles. » (1999:269-270).

Le sociologue Wilfried Spohn s'est récemment élevé contre la tendance qui consiste à généraliser l'ethnicisme à toutes les revendications identitaires et collectives (comme les nationalismes ou communautarismes) d'autant plus lorsqu'elles se manifeste hors du monde occidental ou à sa marge $(2003: 266)$ : comme si, hors des frontières mouvantes d'un Occident engagé dans la modernité et ses avatars (postmodernité, ultra-modernité, surmodernité...), ou initiateur d'une mondialisation qui lui échappe, le reste des nations était restée figé à l'ère de "l'ethnie ». Andrew M. Greeley avait déjà, à partir de l'étude des " minorités " nord-américaines, qualifié l'ethnicité de "survivance des formes antérieures de la vie sociale et de la solidarité traditionnelle dans un monde bureaucratique » $(1974$ :27). Anthony Smith a poursuivi cette ligne d'argmentation en affirmant que l'ethnie est le « cœur » (core) " prémoderne » de l'identification collective, avant de laisser place, dans la 
perspective d'une substitution historique, à la nation, une forme territorialisée d'identification (nationale et nationaliste) : ethnie et nation sont ainsi deux produits différents de l'Histoire (1991). Stuart Hall oppose à ces conceptions disjonctives l'idée d'une continuité de l'ethnicité, mais dont les formes contemporaines procèdent de logiques particulières, différentes des « logiques classiques » ou «traditionnelles » de l'identité : des reformulassions identitaires qui ne peuvent plus se fonder sur la stabilité, la continuité et la "solidité » des bases traditionnelles de l'identité, érodées par la modernité (The local and the global, 1991 : 44-45). Martin Albrow tranche finalement le débat en reconnaissant les deux modes d'identification («moderne » et « traditionnels ») mais refuse de les situer sur un plan de sucessivité historique : ils se manifestent au contraire sur un plan de coexistence, et leurs rapports peuvent prendre la forme d'une complémentarité ou d'une opposition. Albrow préfère parler d'ethnicité que d'ethnie (même si Smith considère l'ethnie comme un processus plutôt que comme une forme stable), et rejette l'argument évolutionniste : le label ethnique n'est pas une forme « prémoderne " mais " alternative » à l'identification nationale. C'est en effet l'affirmation d'une identité culturelle énoncée en réaction - souvent sous la forme d'une résistance aux contraintes imposées par les États-nations ou par les réseaux transnationaux, c'est-à-dire, par la mondialisation.

\section{ReMARQUeS CONCLUSIVES}

Si la nouvelle version, proposée par Tomlinson, ne résout pas la question de l'ontologie de la mondialisation mais nourrit simplement, après celui de la " modernité », les innombrables " mythes » qui peuplent la pensée des sciences de l'homme (Argyrou, 2003), elle a au moins le mérite de rappeler que les identités culturelles « en mouvement » ne sont pas nouvelles, mais que, dans le contexte actuel, elles se constituent en opposition ou en résistance aux forces d'une certaine mondialisation, que leur institutionnalisation se maintient dans le rapport entre local et le global, et, de manière plus générale, dans une amplification de la signification des postures identitaires. La mondialisation y apparaît moins par défaut que par excès d'identité, et ainsi s'explique conjointement le recours à des réinventions historiques ou généalogiques, et la régénération du concept d'ethnogenèse. Mais si le débat s'arrêtait là, la singularité même du concept d'ethnogenèse disparaîtrait purement et simplement avec ces réflexions générales autour de la mondialisation. Ce qui importe, semble-t-il, c'est au contraire d'examiner une ultime fois la portée de ce concept.

Il semble en effet que la notion d'ethnogenèse a, en quelque sorte, suivi très précisément les vicissitudes sémantiques et conceptuelles de l'ethnie - ethnie qui, partant de conceptions essentialistes, s'est trouvée vidée de son essence (posture primordialiste) avant d'être réhabilitée sous une forme dynamique et contextuelle (posture instrumentaliste). Il en va de même pour l'ethnogenèse qui a pour origine (et pour usage encore 
courant) un processus objectivité par l'analyse scientifique (la manière dont un peuple se constitue dans l'histoire...) mais par un glissement des questionnements autour de son ontologie (et de ses mécanismes) dans le domaine strictement académique, se retrouve actuellement comme relevant d'usages et d'instrumentalisations dans les sociétés globales. Déréifiée dans le champ académique, l'ethnogenèse est ainsi devenue un concept d'usage social, dont il y a fort à parier, si le caractère abscons du terme ne rebutait pas les acteurs de la scène politique, qu'il devienne un emblème aussi mobilisateur et controversé de l'époque actuelle, que l'est la mondialisation. Ce glissement de l'ontologie à l'instrumentalisation est aussi celui de l'histoire à la généalogie, ce passage du récit, par la science, de la constitution et des transformations historique des collectifs humains, et leur assigne ainsi une identité " objective ", à celui que les collectifs font de leur propre histoire et se confèrent ainsi une identité, construite dans une contemporanéité complexe, avec une profondeur historique qui en légitime l'origine et la forme.

\section{Bibliographie}

Albrow Martin, The Global Age, Stanford, Stanford U. P., 1997

Appadurai Arjun, « Disjuncture and Difference in the Global Cultural Economy », Theory, Culture \& Society, vol. 7, 1990, réédité sous le même titre in Featherstone Mike (dir.), Global Culture. Nationalism, Globalization and Modernity, $8^{\mathrm{e}}$ édition, Londres, Thousands Oaks, New Delhi, Sage Publications, 1999 [1990], p. 295-310.

- "Grassroots : Globalization and the Research Imagination ", in Appadurai Arjun (dir.), Globalisation, Durham, Londres, Duke U.P., 2001, p. 1-21.

Argyrou Vassos, " "Reflexive modernization" and other mythical realities ", Anthropological Theory, 2003, 3 (1), p. 27-41.

Bartolomé Miguel Alberto, "Les habitants du "désert" : génocide, ethnocide, et ethnogenèse en Argentine ", Les Cahiers Amérique Latine, Histoire et Mémoire, 10, 2004.

Bauman Zygmunt, "Identity in the globalising world ", Social Anthropology, 20019 (2), p. 121-129.

BERKING Helmut. " "Ethnicity is Everywhere" : On Globalization and the Transformation of Cultural Identity ", in ScHUERKENS U. (dir.), Global Forces and Social Life-Worlds. Social Transformations, Londres, Sage, 2004, p. 51-66.

Boccara Guillaume, Guerre et ethnogenèse Mapuche dans le Chili colonial, Paris, L'Harmattan, 1999.

Bromley $\mathrm{Yu}$, "On the Typology of Ethnic Communities », in Holloman Regina \& Ariutiunov Sergei (dir.), Perspectives on Ethnicity, La Haye-Paris, Mouton, 1978, p. 15-21.

- " The objetc and the subject-matter of ethnography " in GELLNER Ernest (dir.) Soviet and Western Anthropology, Londres, Duckworth, 1980, p. $152-160$. 
Curta Florin, "Pots, Slavs and "Imagined Communities" : Slavic Archeologies and the History of the Early Days ", European Journal of Archeology, 4 (3), 2001, p. 367-384.

Dragaze T., "The place of "ethnos" in Soviet Anthropology " in Gellner Ernest (dir.) Soviet and Western Anthropology, Londres, Duckworth, 1980, p. 161-170.

DRUMmOND Lee, "Review of "Ethnic Identity" : Strategies of Diversity ", American Ethnologist, 10 (4), nov. 1983, p. 802-803.

Duelke Britta, "No Matter That Modern World" - An Aboriginal Approach to a Polychronic Past. A Case Study from Northern Australia " in Ton Otto \& Pedersen Poul (dir.), Tradition and Agency. Tracing Cultural Continuity and Invention, Aarhus (Danemark), Aarhus U. P., 2005, p. 267-290.

Dunaway W. A., "Ethnic Conflict in the Modern Word-System : The Dialectics of Counter-Hegemonic Resistance in an Age of Transition ", Journal of World-Systems Research, IX (I), winter 2003, p. 3-34.

Fenton S., «Beyond Ethnicity. The Global Comparative Analysis of Ethnic Conflicts ", International Journal of Comparative Sociology, vol. 45 (3-4), 2004, p. 179-194.

Flannery W P., Reise S. P. \& Jiajuan Yu, « An Empirical Comparison of Acculturation Models ", Personality and Social Psychology Bulletin, 27 (8), 2001, p. 1035-1045.

Friedman J. "Cultural Logics and the World-System. A Sketch », Theory, Culture \& Society, vol. 5, 1988, p. 447-460.

GEMDev, Mondialisation, les mots et les choses, Paris, Karthala, 1999.

Giddens Anthony, Les conséquences de la modernité, Paris, L'Harmattan, 1994.

Glazer Nathan \& Moynihan Daniel. P. (dir.), Ethnicity, Theory and Experience, Cambridge, Harvard U. P, 1975.

Greeley Andrew M., Ethnicity in the United States. A Preliminary Reconnaissance, New York, John Wiley \& Sons, 1974.

HALl Stuart, «The local and the global : Globalization and Ethnicity »; " Old and new identities, old and new ethnicities » in KING Anthony (dir.), Culture, Globalization, and the World-System, Londres, MacMillan, 1991, p. 19-39 et 41-68.

Hoppenbrouwers Peter, "Such Stuff as Peoples are Made of : Ethnogenesis and the constitution of Nationhood in Medieval Europe ", The Medieval Journal History, 9 (2), 2006, p. 195-242.

Killbrow Ann E., Biblical Peoples and Ethnicity : An Archeological Study of Egyptians, Canaanites, Philistines and Early Israel, 1300-100 BCE, Atlanta, sBL, 2005.

KIM Young Yun, «From Ethnic to Interethnic. The Case of Identity Adaptation and Transformation ", Journal of Language and Social Psychology, 25 (3), 2006, p. 283-300.

LAMINE Anne-Sophie, "L'ethnicité comme question sociologique ", Archives de sciences sociales des religions, 131-132, 2005, p. 189-197.

Lenclud Gérard, Bonte Pierre \& IzArd, Michel, " Les anthropologues russes et soviétiques " in Bonte Pierre \& IzARD Michel (dir.), Dictionnaire de l'ethnologie et de l'anthropologie, Paris, PUF, 2002, p. 730-732. 
LÉvi-Strauss Claude, Race et Histoire, Paris, Denoël/Unesco, 1955.

Luca Nathalie \& Burrell Jean, "Borrowings go Round and Round. Transcending Borders and Religious Flexibility ", Diogenes, 47, 1999 , p. 3-10.

MARTin Adrian, " Environmental Conflict betterave Refugees and Host Countries », Journal of Peace Research, 43 (3), 2005, p. 329-346.

Mingming Wang, "The Third Eye. Towards a Critique of "Nativist Anthropology" ", Critique of Anthropology, 22 (2), 2002, p. 149-174.

Montalvo Frank. F. \& CodinA G. Edward, "Skin Colour and Latinos in the United States », Ethnicities, vol 1 (3), 2001, p. 321-341.

Morgan George, "Autochtonous Australian Syncretism ", Current Sociology, vol. 51 (3/4), 2003, pp. 433-451.

PREYer Gerhard \& Bös Mathias, « Borderlines in Time of Globalization ", s.d., disponible sur le site http://www.protosociology.de/ Download/P15-Introduction.pdf

Roosens Eugen E., Creating Ethnicity. The Process of Ethnogenesis, Newbury Park, Sage, 1989.

SAnsone Livio, Blackness without Ethnicity. Constructing Race in Brazil, Londres, Palgrave MacMillan, 2003.

Scarduelli Pietro, "Padanian Identity. Ethnogenesis as a Political Strategy » in Ton Otto \& Pedersen Poul (dir.), Tradition and Agency. Tracing Cultural Continuity and Invention, Aarhus (Danemark), Aarhus U. P., 2005, p. 139-155.

SCHNAPPER Dominique, "Renouveau ethnique et renouveau religieux dans les démocraties providentielles ", Archives des sciences sociales des religions, 131-132, 2005, p. 9-26.

Smith Anthony, National Identity, Harmondsworth, Penguin, 1991.

Spohn Willfried, «Multiple Modernity, Nationalism and Religion : A Global Perspective », Current Sociology, 51(3/4), p. 265-286.

Spybey Tony, Globalization and World Society, Cambridge, Polity Press, 1996.

TAYLOR Christopher C. "Dual Systems in Rwanda. Have they ever existed?", Anthropological Theory, 4 (3), 2004, p. 353-371.

Thornike Gordadze, "La Géorgie et ses "hôtes ingrats" ", Revue Critique Internationale, $\mathrm{n}^{\circ} 10$, Juin 2001, disponible à l'URL : http://www. ceri-sciences-po.org/publica/critique/article/ci10p161-176.pdf

Tiryakian Edward A, "Introduction : Comparative Perspectives on Ethnicity and Ethnic Conflicts ", International Journal of Comparative Sociology, vol 45 (3-4), 2004, p. 147-159.

TomLInson John, Globalization and Culture, Chicago, The U. of Chicago Press/Polity Press, 1999.

Turner Thomas, "'Batetela', 'Baluba', 'Basonge'. Ethnogenesis in Zaire », Cahier d'Études Africaines, 33 (132), 1993, p. 587-612.

VAN BLy Martha C., "Globalization and the Rise of one Heterogeneous World Culture. A Microperspective of a Global Village », International Journal of Comparative Sociology, 48 (2-3), 2007, p. 234-256.

Weber Max, "Les groupes ethniques », dans Économie et Société, Paris, Plon, 1971. 